\title{
GATA5 CpG island hypermethylation is an independent predictor for poor clinical outcome in renal cell carcinoma
}

\author{
INGA PETERS $^{1}{ }^{1}$, KAI GEBAUER ${ }^{1}$, NATALIA DUBROWINSKAJA ${ }^{1}$, FARANAZ ATSCHEKZEI ${ }^{1}$, \\ MARIO W. KRAMER ${ }^{1}$, JOERG HENNENLOTTER ${ }^{4}$, HOSSEIN TEZVAL ${ }^{1}$, MAHMOUD ABBAS $^{2}$, RALPH SCHERER $^{3}$, \\ AXEL S. MERSEBURGER ${ }^{1}$, ARNULF STENZL ${ }^{4}$, MARKUS A. KUCZYK ${ }^{1}$ and JUERGEN SERTH ${ }^{1}$ \\ Departments of ${ }^{1}$ Urology and Urologic Oncology, ${ }^{2}$ Pathology and ${ }^{3}$ Biometry, Hannover Medical School, \\ D-30625 Hannover; ${ }^{4}$ Department of Urology, Eberhard-Karls University, D-72076 Tuebingen, Germany
}

Received November 3, 2013; Accepted December 2, 2013

DOI: $10.3892 / o r .2014 .3030$

\begin{abstract}
Transcriptional inactivation and $\mathrm{CpG}$ island (CGI) methylation of GATA transcription factor family members GATA3 and GATA5 have been reported for a few types of human cancer. Whether high-density CGI methylation of GATA3 or GATA5 is associated with the clinical course of patients with renal cell cancer (RCC) has not been clarified. Quantitative methylation-specific PCR assays were carried out to analyze 25 tumor cell lines including 6 RCC lines and 119 RCC and 87 adjacent normal tissues for the presence of densely methylated sequences. Methylation values were statistically compared with clinicopathological and recurrence-free survival (RFS) data for patients. Comparison of GATA3 and GATA5 methylation in different tumor cell lines revealed a marker-specific methylation characteristic with high and frequent signals for both methylation marks in RCC lines. GATA3 and GATA5 CGI relative methylation levels were found to be strongly associated with the state of metastasis $(\mathrm{P}=0.003$ and $\mathrm{P}<0.001$, respectively) and advanced disease $(\mathrm{P}=0.024$ and $\mathrm{P}<0.001$, respectively). Moreover, an independent decrease in RFS in Cox proportional hazard analysis was found for tumors exhibiting high GATA5 methylation $(\mathrm{P}<0.001$, hazard ratio, 19.3; 95\% confidence interval, 4.58-81.6). Epigenetic alterations in GATA family members may be associated with aggressive tumor phenotypes in $\mathrm{RCC}$, and in the case of GATA5, may serve as a new independent molecular marker for aggressiveness and disease progression.
\end{abstract}

\section{Introduction}

Renal cell carcinoma ( RCC) is the tenth most common cancer in men worldwide (1) and the third most common genitourinary

Correspondence to: Dr Juergen Serth, Department of Urology and Urologic Oncology, Hannover Medical School, Carl-NeubergStrasse 1, D-30625 Hannover, Germany

E-mail: serth.juergen@mh-hannover.de

Key words: GATA3, GATA5, renal cell cancer, DNA hypermethylation, survival, prognosis tumor. The use of targeted therapies has improved treatment of metastatic RCC, but survival remains significantly decreased in late-stage or metastatic RCC patients (2).

The molecular carcinogenesis of clear cell renal cell carcinoma (ccRCC) includes von Hippel-Lindau gene alterations as gatekeeper mutations that are followed by additional genetic changes for full development of the cancer (3). In view of the epigenetic progenitor cancer model, such mutations may be substituted by epigenetic alterations that cause gene silencing and thus contribute to the accumulation of epigenetic and genetic alterations, as has been found for several human malignancies (4). Indeed, a considerable number of loci undergoing DNA methylation have been identified in ccRCC at a high frequency. For example, the secreted frizzled-related protein (SFRPI) and RAS-associated domain family $1 \mathrm{CpG}$ island (CGI) hypermethylation have been found in 34-68\% and $28-76 \%$ of RCCs, respectively (5-7). Hypermethylation of the $S C U B E 3$ gene is associated with clinicopathological parameters and poorer survival (8). A genome-wide CGI methylation analysis by Ricketts et al (9) showed that CGI hypermethylation of several genes (including SLC34A2 in 63\%, OVOL1 in $40 \%$, DLEC in 20\%, TMPRSS2 in 26\%, SST in $31 \%$ and BMP4 in $35 \%$ of RCC) is associated with transcriptional silencing, reactivation after demethylation in $\mathrm{RCC}$ cell lines and downregulation of expression in $\mathrm{RCC}$.

Recently, we identified GATA5, a member of the GATA transcription factor family (GATA1 to GATA6), as a new target for CGI hypermethylation in RCC, also demonstrating a statistical association with disease progression and decreased survival. However, since combined bisulfite restriction analysis detection was applied for methylation detection, only site-specific average methylation could be assessed (10). Heterogeneous methylation as determined in the CGI of GREM1 in RCC (11) may lead to varying statistical associations with clinicopathological parameters; thus, our previous findings of GATA5 CGI methylation as a potential prognosticator for RCC would be strengthened if another GATA5 methylation locus could be identified to demonstrate association with an unfavorable prognosis. Detecting highly methylated sequences located in a different subregion of the GATA5 CGI would provide further evidence for a crucial role of GATA5 in RCC progression. 
In addition, comparing expression and methylation data from public databases (12), we noted that GATA3, as a member of the GATA transcription factor family, might also represent a potential target for CGI hypermethylation. The GATA1, GATA2 and GATA3 members of the GATA transcription factor family are functionally involved in cellular lineage determination (13) while the GATA4, GATA5 and GATA6 are mainly involved in epithelial differentiation and are suggested to play a critical role in tumorigenesis of cancer with endoor mesodermal origins (13). Furthermore, both mechanisms exhibit extensive changes in neoplastic development in different cancer types (14) and loss of GATA3 expression in breast cancer patients has been significantly associated with poor clinical outcome and advanced tumor disease (15). Comparing normal and tumor renal tissues, decreased GATA3 protein and mRNA expression levels have already been observed, supporting the hypothesis that GATA3 may be epigenetically silenced in RCC (16).

To clarify the relevance of GATA3 and GATA5 methylation in RCC, we measured CGI methylation of both genes in normal human primary tubule epithelial cells and in renal tumor cell lines, as well as in renal cancer tissues and a subset of paired adjacent normal tissues, using quantitative methylation-specific PCR (qMSP). We found that higher methylation is more likely to be found in tumors of patients with advanced and metastatic disease and in case of GATA5 is also associated with poorer survival of RCC patients.

\section{Materials and methods}

Tissue specimens. Cross-sectional analyses were conducted on 119 RCC samples and 87 samples from paired histologically normal-appearing tissues, i.e., adjacent normal renal tissue. Tissue samples were collected from patients who had undergone radical or nephron-sparing nephrectomy and stored as previously described (17). TNM classification of all tissues was evaluated according to the Union for International Cancer Control 2010 classification, and grading was assessed as previously described $(18,19)$. Localized RCC was defined as $\mathrm{pT} \leq 2$, lymph node (N) and metastasis (M) negative (N0 and M0), and a grading $(\mathrm{G})$ of 1 and 1-2. Advanced tumors were classified as $\mathrm{p} \geq \mathrm{T} 3$ and/or lymph node positive $(\mathrm{N}+)$, positive for distant metastasis (M+) or G2-3 and G3. Time to disease recurrence was designated as the point at which patients had either a local recurrence or a synchronous/metachronous metastasis as detected by computerized tomography scan. The local ethics committee approved sample collection, and informed consent was obtained from each patient. Clinical and histopathological parameters of tissues are summarized in Table I. Purchase, culturing, storage and identity control of cell lines and primary cells were carried out as previously described (17).

Isolation of DNA and bisulfite conversion. DNA was extracted from frozen tissue sections using a standard phenol/chloroform extraction method. Bisulfite conversions and histopathological examination of control sections were conducted as previously reported (20).

Quantitative methylation-specific real-time PCR analysis of GATA3 and GATA5 CGI methylation. Methylation analyses
Table I. Clinicopathological data of patients.

\begin{tabular}{lcc}
\hline Clinicopathological parameters & GATA3 $(\%)$ & GATA5 (\%) \\
\hline Cases in total (all RCC) & $119(100)$ & $109(100)$ \\
Histology & & \\
ccRCC & $86(72)$ & $78(72)$ \\
papRCC & $24(20)$ & $22(20)$ \\
Chromophobe/mixed RCC & $5(4)$ & $5(5)$ \\
Not classified & $4(3)$ & $4(4)$ \\
Gender & & \\
Female & $42(35)$ & $37(34)$ \\
Male & $77(65)$ & $72(66)$ \\
Age (years) & & \\
Median & $65(55)$ & $65(60)$ \\
Tumor size & & \\
In diameter (cm) & 4.6 & 4.5 \\
Primary tumor classification & & \\
pT1 & $11(9)$ & $11(10)$ \\
pT1a & $35(29)$ & $32(29)$ \\
pT1b & $19(16)$ & $19(17)$ \\
pT2 & $8(7)$ & $6(6)$ \\
pT3 & $5(4)$ & $4(4)$ \\
pT3a & $11(9)$ & $8(7)$ \\
pT3b/c & $25(21)$ & $24(22)$ \\
pT4 & $1(1)$ & $1(1)$ \\
Not known & $4(3)$ & $4(4)$ \\
Ly & &
\end{tabular}

Lymph node status

$\begin{array}{lrr}\mathrm{N} 0 & 104(87) & 96(88) \\ \mathrm{N}+ & 15(13) & 13(12)\end{array}$

Metastasis classification

M0

$92(77) \quad 85(78)$

$\mathrm{M}+$

$27(23)$

Grade

Low risk group

$\begin{array}{lll}\text { G1 } & 23(19) & 22(20) \\ \text { G1-2 } & 16(13) & 14(13) \\ \text { G2 } & 60(50) & 57(52)\end{array}$

High risk group

$\begin{array}{lrr}\text { G2-3 } & 9(8) & 7(6) \\ \text { G3 } & 11(9) & 9(8)\end{array}$

Localized disease

pT $\leq 2$, N0, M0 and G1; G1-2 63 (53) 58 (53)

Advanced disease

$\mathrm{pT} \geq 3$ and/or $\mathrm{N}+, \mathrm{M}+$ or $\mathrm{G} 2-3 ; \mathrm{G} 3 \quad 55$ (46) $\quad 50$ (46)

Not known 1 (1) 1 (1)

Paired samples
All RCC
$87(73)$
$77(71)$
ccRCC
$66(55)$
$57(52)$

ccRCC, clear cell renal cell carcinoma; papRCC, papillary renal cell carcinoma. 


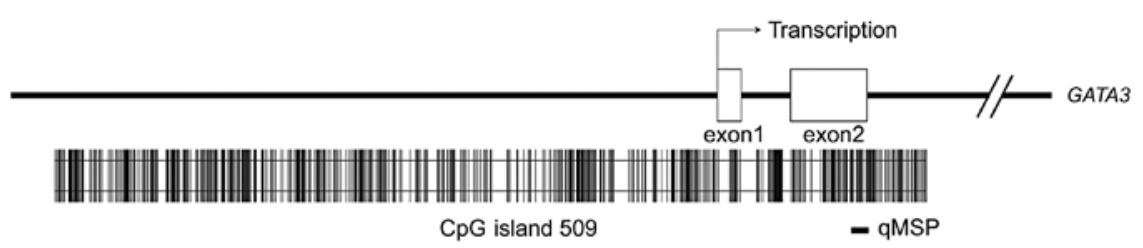

A-b chromosome 20q13.33

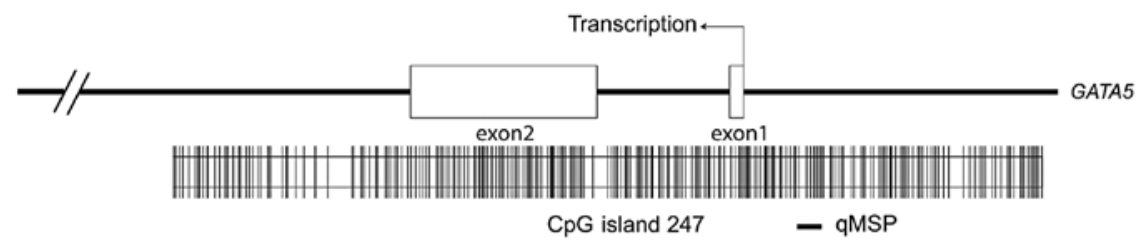

B-a
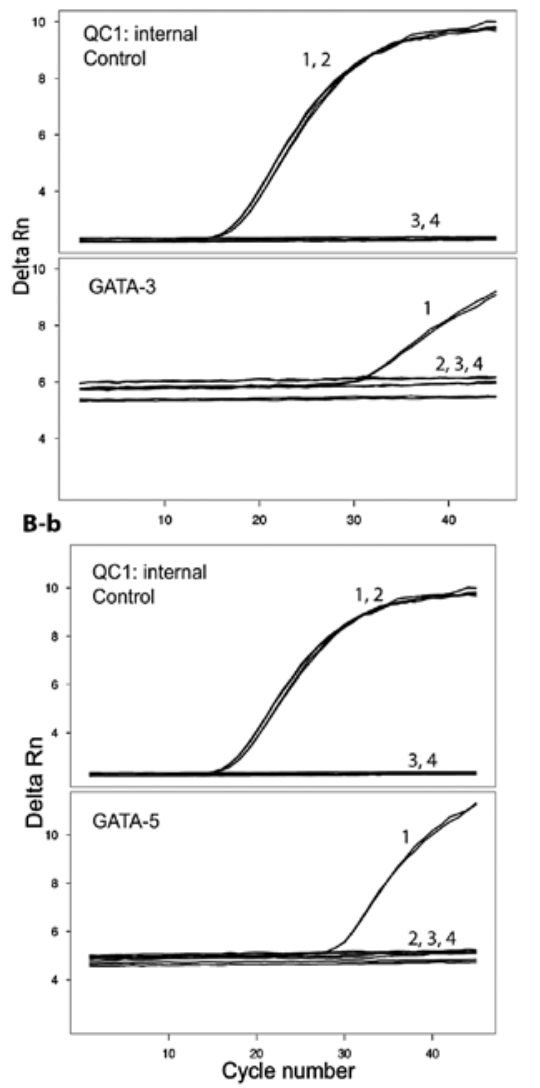

C-a
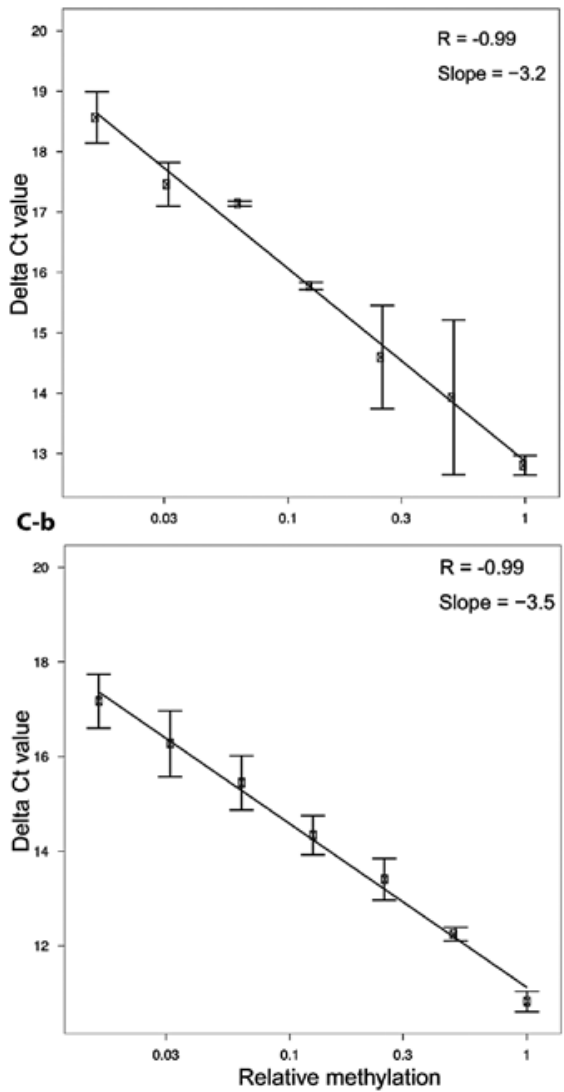

Figure 1. Description of investigated CpG islands of GATA3 and GATA5 and assay controls. (A-a) Structure of the GATA3 CGI locus and location of the qMSP assay relative to the transcription start site. GATA3 is located on chromosome 10p14. CpG sites are illustrated with vertical lines within the CpG island. Information refers to UCSC Genome Browser database and GenBank (12,22). (A-b) GATA5 structure and location of the qMSP assay relative to the transcription start site. Vertical lines represent CpG sites within the CpG island. Base positions refer to the GRCh37/hg19 annotation in the UCSC Genome Browser and GenBank (12,22). (B) Primary data of quantitative methylation-specific and control PCR measurements in methylated control DNA (1); unmethylated control DNA (2); unconverted DNA (3); and a blank control (4) for GATA3 (a) and GATA5 (b) analysis. (C) Normalized GATA3 (a) and GATA5 (b) assay threshold values $(\mathrm{Ct})$ for a 2 -fold dilution series of the methylated control in non-methylated control DNA for determination of assay linearity and efficiency.

of bisulfite-treated genomic DNA for CGI methylation of GATA3 and GATA5 was performed by quantitative real-time fluorimetric 5' exonuclease methylation-specific PCR assays. Methylation analysis was carried out as described elsewhere (21). The qMSP-specific primers 5'-TGTATCGGGACGGA ATCGTT-3' (forward) and 5'-ACGCGCGCTCTAACCCTT-3' (reverse) as well as the $\operatorname{Taqman}^{\circledR}$ probe 5'-FAM-AAATAT
AACCGCGACTCCTACCAATTCATTCG-BHQ-3' were designed using Beacon Designer ${ }^{\mathrm{TM}}$ software (Premier Biosoft, Palo Alto CA, USA). Intra-CGI location of both qMSP assays, designed within an area of high GC percentage, is shown in Fig. 1A-a (GATA3) and in Fig. 1A-b (GATA5). Table II shows the base positions of investigated $\mathrm{CpG}$ sites in the corresponding CGI referenced in the USCS Genome 
Table II. Detailed chromosomal information of GATA3 and GATA5.

\begin{tabular}{lcc}
\hline & GATA3 & GATA5 \\
\hline Chromosome & $10 \mathrm{p} 14$ & $20 \mathrm{q} 13.33$ \\
GeneID & 2625 & 140628 \\
CpG Island & 509 & 247 \\
No. of CpG sites & $8091375-8098329$ & $61049362-61051897$ \\
Base position (bp) & $8097735, \sim 744, \sim 750, \sim 796$, & $61051188, \sim 210, \sim 223, \sim 232$, \\
bp of CpG sites investigated by qMSP & $\sim 801, \sim 811, \sim 831, \sim 849$ & $\sim 236, \sim 241, \sim 253, \sim 255, \sim 262$ \\
& & \\
\end{tabular}

Chromosomal information and base position (bp) location of GATA3 and GATA5 qMSP relevant CpG sites. Information refers to the UCSC Genome Browser annotation GRCh37/hg 19.
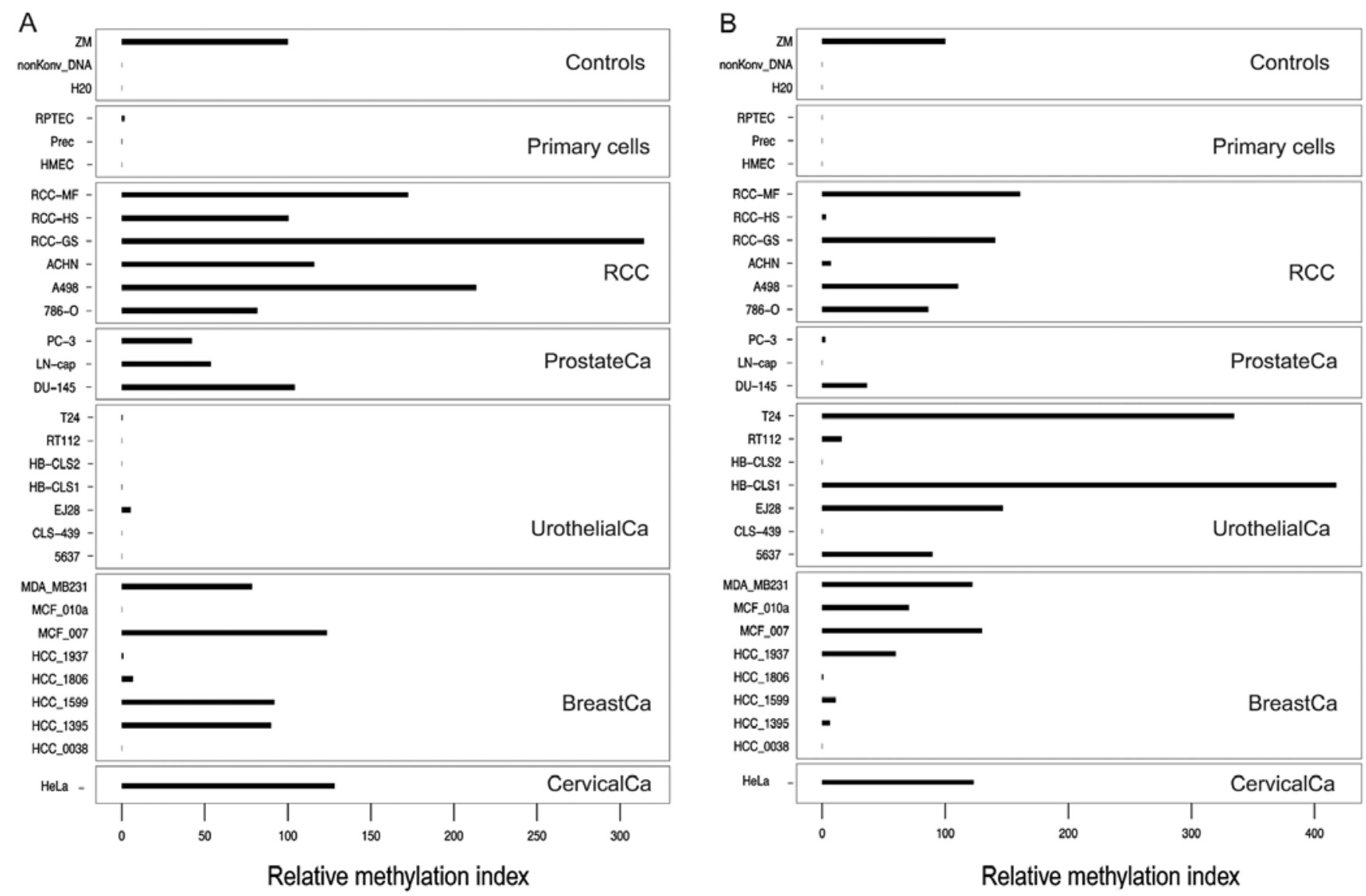

Figure 2. Measurement of relative methylation in different cancer cell lines. Levels of relative methylation values in cancer cell lines and normal primary cells for (A) GATA3 and (B) GATA5.

Browser $(12,22)$. Real-time PCR was carried out in duplicate using a FasTrans automatic Liquid Handling System (Analytik Jena, Jena, Germany) and an ABI 7900HT (Life Technologies, Foster City, CA, USA) in 384-well plates as previously reported (17). An experimenter who was blinded to type, order and clinicopathological status of samples carried out measurements.

Calculation of relative methylation indices and statistical analysis. Calculation of the relative degree of methylation was based on the method of Weisenberger et al, recently described in detail $(21,23)$. Statistical analyses and definition of the cut-off value for dichotomization used in survival analysis were also carried out as previously described (17).

For univariate statistical analyses, all groups were dichotomized according to their clinicopathological parameters, i.e., localized (Loc.) vs. advanced (Adv.) disease, metastasis negative (M0) vs. positive $(\mathrm{M}+)$, lymph node metastasis-negative vs. lymph node metastasis-positive $(\mathrm{N} 0 / \mathrm{N}+)$, and low-grade (G1, G1-2) vs. high-grade (G2-3, G3) tumors. 
Table III. Statistical analyses of GATA3 and GATA5 CGI methylation and correlation with clinicopathological parameters in paired t-test and univariate logistic regression analysis.

GATA3
P-value
P-value

GATA5

$\operatorname{adN} / \mathrm{TU}$

all RCC

0.006

$<0.001$

ccRCC

0.001

$<0.001$

Univariate logistic regression analysis

OR $(95 \% \mathrm{CI})$

$0.77(0.63-0.94)$

$1.32(1.04-1.68)$

$1.16(0.89-1.50)$

0.277

0.003

0.179

$1.59(1.05-2.43)$

$1.38(0.86-2.20)$

0.658

0.542

$1.06(0.82-1.37)$

$0.92(0.68-1.21)$

0.187

0.572
$1.36(0.86-2.14)$

$1.17(0.68-2.01)$
OR $(95 \% \mathrm{CI})$

$0.80(0.67-0.96)$

0.015

1.55 (1.29-1.88)

$1.46(1.19-1.80)$

$<0.001$

1.65 (1.29-2.11)

$1.64(1.23-2.17)$

$<0.001$

1.47 (1.14-1.88)

1.54 (1.11-2.14)

0.003

$1.32(1.03-1.68)$

0.03

$1.15(0.85-1.56)$

adN, adjacent normal tissue; TU, tumor tissue; ccRCC, clear cell renal cell carcinoma; papRCC, papillary renal cell carcinoma; OR, odds ratio; CI 95\%, confidence interval. ${ }^{a}$ Localized tumor is $\mathrm{pT} \leq 2$, lymph node (N) and metastasis (M) negative (N0/M0) and grading (G) G1 and G1-2. Advanced tumor is $\mathrm{pT} \geq 3$ and/or $\mathrm{N}+, \mathrm{M}+$ or G2-3 and G3. bow grade tumor (G1 and G1-2). High grade tumor (G2-3 and G3).

\section{Results}

Measurement of technical controls and analysis of GATA3 and GATA5 CGI methylation in human normal cells and cancer cell lines. The specificity of the GATA3 and GATA5 qMSP analyses was evaluated by duplicate measurements of converted methylated (M), converted non-methylated (U) and non-converted DNA control samples. For U and non-converted DNA samples, we exclusively measured $\mathrm{Ct}$ values of 45 (undetermined) whereas the $\mathrm{M}$ sample demonstrated $\mathrm{Ct}$ values of $\sim 32$ for GATA3 (Fig. 1B-a) and Ct values of 29 for GATA5 (Fig. 1B-b). None of the control or CGI-specific qMSP assays gave signals for non-converted DNA, thus demonstrating that only methylated and converted DNA was detected. PCR efficiency and linearity of the methylation detection assays were assessed using a log dilution series of the $\mathrm{M}$ control within the U control DNA and adjusting for constant total converted DNA amount in PCR reactions. Linear regression analyses demonstrated a slope of $\Delta \mathrm{Ct}=-3.3$ per 10 -fold dilution and a coefficient of correlation of $\mathrm{r}=-0.99$ for both genes $(\mathrm{P}=0.001)$, indicating linearity of the assays (Fig. 1C-a and 1C-b).

We assessed whether the GATA3 and GATA5 qMSP assays are capable of methylation detection in normal human primary tubule epithelial cells and in cancer cell lines, each respectively used as a proxy for normal tissues and localized and metastatic human cancers of other origin (kidney, prostate, bladder, breast and cervical cancer cell lines), which in part have already been reported to demonstrate tumor specific hypermethylation. Methylation for GATA3 was found in 5/8 (63\%) breast cancer cell lines, as expected from previous reports describing GATA3 methylation in breast cancer tissue samples. Notably, all 6 renal cancer cell lines showed high relative methylation indices while normal primary cells from kidney (RPTEC), prostate cancer, and mammary tissues demonstrated low or undetectable methylation (Fig. 2A). Similarly, GATA5 CGI methylation was not detectable or was low in normal primary cells but demonstrated higher relative methylation indices only for 4/6 renal cancer cell lines (Fig. 2B).

GATA3 and GATA5 CGI is hypermethylated in RCC. Comparison of GATA3 and GATA5 methylation in matched tumor (TU) and adjacent normal (adN) tissues demonstrated tumor-specific hypermethylation (Fig. 3A and B). Using the paired t-test for statistical analysis (Table III), we found significant differences for GATA3 methylation in the RCC tissue groups $(\mathrm{P}=0.006)$ as well as in the ccRCC subset $(\mathrm{P}=0.001)$. The corresponding analysis of GATA5 methylation also demonstrated higher methylation in tumor tissues both for the RCC group $(\mathrm{P}<0.001)$ and the ccRCC subset $(\mathrm{P}<0.001)$.

Analysis of GATA3 and GATA5 CGI methylation and association with clinicopathological parameters. Univariate 
A

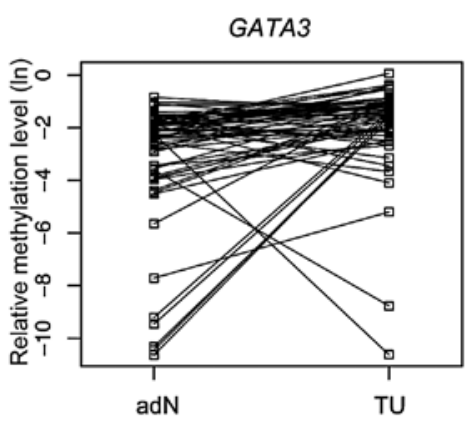

C
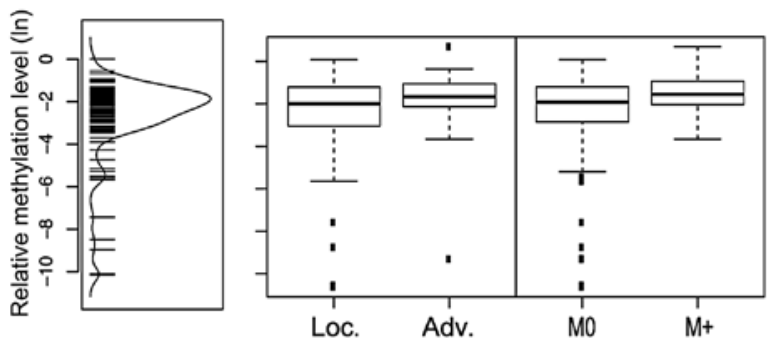

D
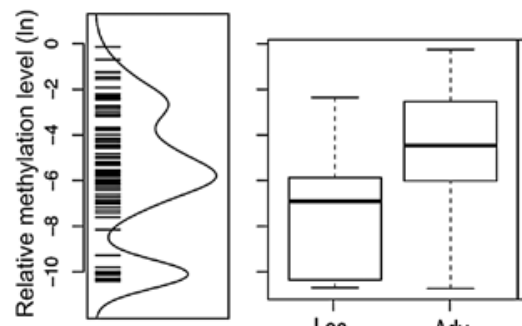

Loc.

Adv

$E$

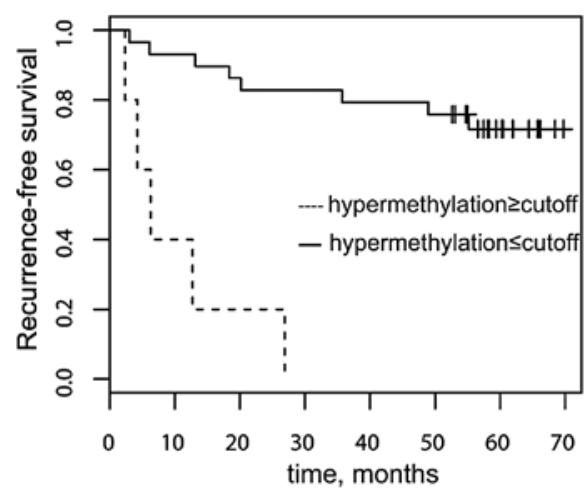

Figure 3. Associations of DNA methylation of GATA3 and GATA5 with clinicopathology and recurrence-free survival. Comparison of the natural logarithms of relative methylation values for (A) GATA3 and (B) GATA5 in adjacent normal (adN) and tumor (TU) tissues of a ccRCC patient cohort (P=0.001, $\mathrm{P}<0.001$ ). (C) Box plot illustration of GATA3 CGI methylation. GATA3 methylation was significantly increased in advanced (Adv.) and metastasized (M+) renal cell cancer patients compared to localized (Loc.) and non-metastasized (M0) disease ( $\mathrm{P}=0.024$ and $\mathrm{P}=0.003$, respectively). Distribution of relative methylation values is illustrated in the Kernel distribution graph. (D) Distribution of GATA5 methylation values in all RCCs in association with clinicopathological parameters: Loc. and Adv. $(\mathrm{P}<0.001), \mathrm{M} 1$ and $\mathrm{M} 0(\mathrm{P}<0.001)$, lymph node status $(\mathrm{NO} / \mathrm{N}+; \mathrm{P}=0.03)$, and high-risk $(\mathrm{G}>2)$ and low-risk $(\mathrm{G} \leq 2)$ grade $(\mathrm{P}=0.003)$. Distribution of relative methylation values is illustrated in the Kernel distribution graph. (E) Kaplan-Meier plot showing the relative recurrence-free survival of clear cell renal cancer patients with GATA5 hypermethylation. Results were dichotomized by a cut-off of -2.447 . The dashed line indicates the patients with relative methylation value higher than the cut-off of -2.447 , demonstrating a significantly decreased recurrence-free survival.

logistic regression analysis (Table III) of dichotomized groups revealed a statistically significant association between methylation of GATA3 and GATA5 CGI with advanced and metastasized RCC disease. Mean methylation for both CGIs
(GATA3 and GATA5) was significantly higher in advanced vs. localized ( $\mathrm{P}=0.024$ and $\mathrm{P}<0.001$, respectively) and in metastasis-negative (M0) vs. metastasis-positive $(\mathrm{M}+)$ tumors $(\mathrm{P}=0.003$ and $\mathrm{P}<0.001$, respectively; Fig. $3 \mathrm{C}$ and $\mathrm{D})$ of the 
Table IV. Uni- and bivariate Cox regression model analysis of GATA5 CGI methylation.

A, Univariate Cox regression analysis of GATA5 CGI methylation and association with recurrence-free survival in patients with clear cell renal cell carcinoma

\begin{tabular}{lrrr}
\hline & P-value & HR & 95\% CI \\
\hline Methylation & $<\mathbf{0 . 0 0 1}$ & 13.0 & $3.57-47.4$ \\
Status of metastasis (M0/M+) & $\mathbf{0 . 0 1 2}$ & 4.07 & $1.36-12.2$ \\
Localized vs. advanced $^{\mathrm{a}}$ & 0.061 & 3.44 & $0.94-12.5$ \\
Grade (low/high) $^{\mathrm{b}}$ & $\mathbf{< . 0 0 1}$ & 8.46 & $2.49-28.7$ \\
Age-Medianc $^{\mathrm{c}}$ & 0.362 & 0.59 & $0.19-1.82$ \\
\hline
\end{tabular}

B, GATA5 CGI methylation analysis in a bivariate Cox regression model and its association with recurrence-free survival

\begin{tabular}{lccc}
\hline & P-value & HR & 95\% CI \\
\hline Methylation & $<\mathbf{0 . 0 0 1}$ & 19.3 & $4.58-81.6$ \\
Status of metastasis (M0/M+) & $\mathbf{0 . 0 0 4}$ & 5.8 & $1.73-19.4$ \\
Methylation & $\mathbf{0 . 0 0 2}$ & 9.55 & $2.36-38.7$ \\
Localized vs. advanced & 0.355 & 1.96 & $0.47-8.23$ \\
Methylation & $\mathbf{0 . 0 4}$ & 5.35 & $1.1-26.1$ \\
Grade (low/high) & 0.09 & 3.80 & $0.80-18.1$ \\
Methylation $_{\text {Age-Medianc }}$ & $<\mathbf{0 . 0 0 1}$ & 29.7 & $5.72-154$ \\
\hline
\end{tabular}

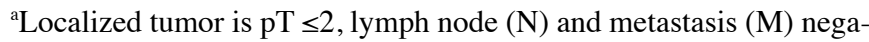
tive. (N0/M0) and grading (G) 1 or $1-2$. Advanced tumor is $\mathrm{pT} \geq 3$ and/or $\mathrm{N}+, \mathrm{M}+$ or G2-3 and G3. bow grade tumor (G1 and G1-2). High grade tumor (G2-3 and G3). 'Values dichotomized by the median of parameter. HR, hazard ratio; CI, $95 \%$, confidence interval. Clinicopathological factors were dichotomized in both regression models.

RCC tissue group. In addition, GATA5 showed a significantly higher CGI methylation status in the high-grade tumor and positive lymph node metastasis $(\mathrm{N}+)$ groups compared to lowgrade tumor tissues $(\mathrm{P}=0.003)$ or negative lymph node status $(\mathrm{P}=0.03$; Fig. 3D). Comparison of CGI methylation of GATA3 and GATA5 in ccRCC and papillary renal cell carcinoma showed significant statistical differences for the mean GATA3 $(\mathrm{P}=0.006)$ and GATA5 $(\mathrm{P}=0.015)$ relative methylation indices observed in both histological entities (Table III).

GATA5 CGI methylation is independently associated with decreased recurrence-free survival. Univariate KaplanMeier and bivariate Cox proportional hazard analysis were conducted to elucidate a possible relationship between GATA3 and GATA5 CGI methylation and recurrence-free survival (RFS) of RCC patients. GATA3 analysis showed no statistical relationship with survival. In contrast, univariate Cox regression analysis revealed GATA5 methylation as a strong parameter in the $\mathrm{RCC}[\mathrm{P}<0.001$; hazard ratio $(\mathrm{HR})=17.8$;
95\% (CI) confidence interval, 4.89-65.1] and ccRCC $(\mathrm{P}<0.001$; $\mathrm{HR}=13$; 95\% CI, 3.57-47.4; Table IVA) tissue groups. The Kaplan-Meier analysis with a calculated optimum cut-off of -2.447 for dichotomization showed that higher CGI methylation of GATA5 is associated with a decreased RFS in patients with ccRCC (Fig. 3E). A pairwise bivariate Cox regression model demonstrated that the GATA5 CGI methylation status remained a significant and strong parameter in the bivariate models when the status of metastasis, advanced tumor disease, grade, and age were considered as co-variables (Table IVB).

\section{Discussion}

GATA1, GATA2 and GATA3 from the GATA transcription factor family are involved in cellular lineage and hematopoietic development while GATA4, GATA5 and GATA6 are involved in epithelial and endodermal differentiations $(13,24)$. GATA proteins have been suggested to play a crucial role in keeping cells in the undifferentiated state (13). Moreover, previous experiments (10) as well as in silico analyses detecting reduced GATA3 and GATA5 mRNA expression levels suggested that GATA3 and GATA5 are potential targets of epigenetic alteration in RCC. The present study has taken a translational approach to investigate the presence and clinical relevance of $\mathrm{CpG}$ island methylation of both genes for RCC.

Tumor cell lines (renal, bladder, prostate and breast cancer) revealed distinct CGI methylation patterns for GATA3 and GATA5 methylation but showed no obvious overall correlation between the epi-alterations. Notably, both methylation markers were frequently observed in kidney-derived tumor cell lines and also demonstrated tumor-specific hypermethylation in RCC in concordance with results for our paired group. The present study identified both genes as candidates with a possible relevance for RCC development. Therefore, our data are in line with a recent functional study demonstrating that methylation-dependent silencing of GATA3 expression is correlated with the loss of transforming growth factor- $\beta$ receptor III and tumorigenesis in ccRCC tissues and cell lines, although its role in disease progression and patient survival remained to be elucidated (25).

Our study revealed that both GATA3 and GATA5 showed a highly significant association between CGI methylation and advanced as well as metastatic RCC. Furthermore, GATA5 CGI methylation exclusively demonstrated a statistical association with grade and lymph node status of the primary tumor. In addition, bivariate Cox regression analysis adjusted for advanced disease, metastatic status, and grade revealed a high and fairly stable HR for GATA5 methylation in the bivariate statistical survival models overall, identifying this epigenetic mark as a new candidate for independent prognosis of decreased RFS.

Although a great number of hypermethylated loci have been identified in RCC (9), to date, only a subset of CGIs has been functionally or clinically characterized. A recent study found that a large portion of clinically relevant epigenetic alterations identified in RCC also exhibit functional changes in kidney cancer (8). Hence, pre-selection of CGIs based on their statistical association with clinical factors could represent an efficient means of narrowing the pool of candidate epi-alterations affecting the onset or course of RCC. Only a 
limited number of methylation-based independent candidate prognosticators including BNC1, COL14A1, SFRP1, SCUBE3, GREMI and $D A L-1 / 4.1 b(6,8,10,11,26)$ have thus far been reported. Therefore, our results identify GATA5 as a new candidate prognosticator gene and suggest its functional relevance in the progression of $\mathrm{RCC}$.

We observed a noticeable difference of approximately two orders of magnitude in the median relative methylation values detected for GATA3 and GATA5 CGIs in tumor compared to adjacent normal renal tissues. Considering that histological assessment of control sections ensured a minimum tumor cell content of at least $50 \%$ and that identical samples have been measured, a variation in tumor cell content as a possible explanation can be ruled out. Instead, we infer that a different methylation characteristic is present in both CGIs, as detected by qMSP specifically measuring completely methylated sequences. Moreover, as the present study only considered single regions within the analyzed CGIs, we cannot rule out that other methylation marks may exist that exhibit significant associations with clinicopathological parameters, bearing in mind that a recent report has shown such intra-CGI variations (11).

The present study identified GATA3 and GATA5 methylation as a common and cancer-specific event in RCC. The association with late-stage disease and for GATA5 with shortened RFS suggests these targets as biomarkers for biological aggressiveness of RCC and, in case of GATA5, as a candidate prognosticator.

\section{Acknowledgements}

We thank Margrit Hepke and Christel Reese for the technical assistance.

\section{References}

1. Jemal A, Bray F, Center MM, Ferlay J, Ward E and Forman D: Global cancer statistics. CA Cancer J Clin 61: 69-90, 2011.

2. Ljungberg B: Prognostic factors in renal cell carcinoma. Urologe A 43 (Suppl 3): S119-S120, 2004.

3. Kaelin WG Jr: The von Hippel-Lindau tumor suppressor gene and kidney cancer. Clin Cancer Res 10: 6290S-6295S, 2004.

4. Feinberg AP, Ohlsson R and Henikoff S: The epigenetic progenitor origin of human cancer. Nat Rev Genet 7: 21-33, 2006.

5. Dahl E, Wiesmann F, Woenckhaus M, et al: Frequent loss of SFRP1 expression in multiple human solid tumours: association with aberrant promoter methylation in renal cell carcinoma. Oncogene 26: 5680-5691, 2007.

6. Morris MR, Ricketts C, Gentle D, et al: Identification of candidate tumour suppressor genes frequently methylated in renal cell carcinoma. Oncogene 29: 2104-2117, 2010.
7. Peters I, Rehmet K, Wilke N, et al: RASSF1A promoter methylation and expression analysis in normal and neoplastic kidney indicates a role in early tumorigenesis. Mol Cancer 6: 49, 2007.

8. Morris MR, Ricketts CJ, Gentle D, et al: Genome-wide methylation analysis identifies epigenetically inactivated candidate tumour suppressor genes in renal cell carcinoma. Oncogene 30: 1390-1401, 2011.

9. Ricketts CJ, Morris MR, Gentle D, et al: Genome-wide CpG island methylation analysis implicates novel genes in the pathogenesis of renal cell carcinoma. Epigenetics 7: 278-290, 2012

10. Atschekzei F, Hennenlotter J, Janisch S, et al: SFRPI CpG island methylation locus is associated with renal cell cancer susceptibility and disease recurrence. Epigenetics 7: 447-457, 2012.

11. van Vlodrop IJ, Baldewijns MM, Smits KM, et al: Prognostic significance of Gremlin1 (GREM1) promoter CpG island hypermethylation in clear cell renal cell carcinoma. Am J Pathol 176: 575-584, 2010.

12. Kent WJ, Sugnet CW, Furey TS, et al: The human genome browser at UCSC. Genome Res 12: 996-1006, 2002.

13. Patient RK and McGhee JD: The GATA family (vertebrates and invertebrates). Curr Opin Genet Dev 12: 416-422, 2002.

14. Chou J, Provot S and Werb Z: GATA3 in development and cancer differentiation: cells GATA have it! J Cell Physiol 222: 42-49, 2010.

15. Abba MC, Nunez MI, Colussi AG, Croce MV, Segal-Eiras A and Aldaz CM: GATA3 protein as a $M U C 1$ transcriptional regulator in breast cancer cells. Breast Cancer Res 8: R64, 2006.

16. Tavares TS, Nanus D, Yang XJ and Gudas LJ: Gene microarray analysis of human renal cell carcinoma: the effects of HDAC inhibition and retinoid treatment. Cancer Biol Ther 7: 1607-1618, 2008.

17. Gebauer K, Peters I, Dubrowinskaja N, et al: Hsa-mir-124-3 $\mathrm{CpG}$ island methylation is associated with advanced tumours and disease recurrence of patients with clear cell renal cell carcinoma. Br J Cancer 108: 131-138, 2013.

18. Sobin LH and Compton CC: TNM seventh edition: what's new, what's changed: communication from the International Union Against Cancer and the American Joint Committee on Cancer. Cancer 116: 5336-5339, 2010.

19. Thoenes W and Storkel S: Pathology of benign and malignant renal cell tumors. Urologe A 30: W41-W50, 1991 (In German).

20. Peters I, Eggers H, Atschekzei F, et al: GATA5 CpG island methylation in renal cell cancer: a potential biomarker for metastasis and disease progression. BJU Int 110: E144-E152, 2012.

21. Weisenberger DJ, Siegmund KD, Campan M, et al: CpG island methylator phenotype underlies sporadic microsatellite instability and is tightly associated with BRAF mutation in colorectal cancer. Nat Genet 38: 787-793, 2006.

22. Lander ES, Linton LM, Birren B, et al: Initial sequencing and analysis of the human genome. Nature 409: 860-921, 2001.

23. Weisenberger DJ, Campan M, Long TI, et al: Analysis of repetitive element DNA methylation by MethyLight. Nucleic Acids Res 33: 6823-6836, 2005.

24. Graf T and Enver T: Forcing cells to change lineages. Nature 462: 587-594, 2009.

25. Cooper SJ, Zou H, Legrand SN, et al: Loss of type III transforming growth factor- $\beta$ receptor expression is due to methylation silencing of the transcription factor GATA3 in renal cell carcinoma. Oncogene 29: 2905-2915, 2010.

26. Yamada D, Kikuchi S, Williams YN, et al: Promoter hypermethylation of the potential tumor suppressor DAL-1/4.1B gene in renal clear cell carcinoma. Int J Cancer 118: 916-923, 2006. 